\title{
O uso da análise funcional na literatura brasileira de terapia comportamental: uma revisão teórico-conceitual
}

\author{
The use of functional analysis in the Brazilian behavior therapy literature: a \\ theoretical-conceptual review
}

\section{El uso del análisis funcional en la literatura brasileña de terapia conductual: una revisión teórico-conceptual}

Maíra Pereira Toscano ${ }^{1}$, Ana Carolina Macchione ${ }^{2}$, Jan Luiz Leonardi ${ }^{3}$

[1] [2] Paradigma - Centro de Ciências e Tecnologia do Comportamento [3] Paradigma - Centro de Ciências e Tecnologia do Comportamento InPBE - Instituto de Psicologia Baseada em Evidências | Título abreviado: Análise funcional na terapia comportamental | Endereço para correspondência: | Email: mairatoscano@gmail. com I doi: 10-18761/PAC.TAC.2019.004 | Nota: Financiamento da pesquisa: recursos próprios. Declaração de conflito de interesse: não há conflito de interesses. 
Abstract: Behavior Analysis investigates, both experimentally and interpretively, the relationships established between the individual and the environment, which is called functional analysis. Although functional analysis is central to the research and the practice of the behavior analyst, there are inconsistencies both in its conceptual characterization and in its use in Brazilian clinical practice. In light of this, this research aimed to investigate the characteristics of functional analyses in the Brazilian behavior therapy literature. In the data collection procedure, 13 cases of typical outpatient clients where functional analyses were drawn or organized in a table were identified. These functional analyses were systematized and analyzed in different categories, relating to responses, antecedents and consequences, how many and what were the terms used and how they were described. The results showed the main features of the use of functional analysis. Reveal conceptual difficulties in transposition of the theoretical knowledge to practice as much as conceptual difficulties, indicating where it is necessary to invest more efforts to expanding the theoretical knowledge and practical training of Brazilian behavioral therapists.

Keywords: functional analysis; contingency analysis; behavior therapy; behavior analysis; clinical psychology.

Resumen: El Análisis de Conducta investiga, tanto de modo experimental como interpretativo, las relaciones establecidas entre individuo y ambiente, que se conoce como análisis funcional. Aunque el análisis funcional sea central en la investigación y en la práctica del analista de conducta, existen inconsistencias tanto en su caracterización conceptual como en su uso en la práctica clínica brasileña. En vista de ello, esta investigación tuvo por objetivo evaluar las características de los análisis funcionales en la literatura brasileña de terapia conductual. Se identificaron en el procedimento de colección de dados 13 casos de clientes típicos atendidos en el ambiente de consultorios en los cuales análisis funcionales fueron diseñadas o organizadas en forma de tabla. Estos análisis funcionales fueron sistematizados y analizados en diferentes categorías, relacionadas con antecedentes, respuestas y consecuencias, cuántas y cuales fueron los términos empleados y cómo los describieron. Los resultados encontrados ilustran las principales características del uso del análisis funcional. Revelan dificultad tanto en la transposición del conocimiento teórico a la práctica, como dificuldades conceptuales, indicando donde es necesario invertir más esfuerzos para la expansión del conocimiento teórico y para la formación práctica de terapeutas comportamentales brasileños.

Palabras-clave: análisis funcional; análisis de contingencia; terapia conductual; análisis del comportamiento; psicología clínica. 
A Análise do Comportamento, ciência fundamentada na filosofia do Behaviorismo Radical, é uma área do conhecimento que tem se dedicado a fornecer explicações dos porquês os seres humanos se comportarem da maneira como o fazem. Para cumprir essa tarefa, a Análise do Comportamento investiga, tanto de modo experimental quanto interpretativo, as relações estabelecidas entre indivíduo e ambiente, o que é comumente chamado de análise funcional (Haynes \& O’Brien, 1990; Skinner, 1953/1965; Sturmey, Ward-Horner, Marroquin \& Doran, 2007; Ulian, 2007; Yoman, 2008).

$\mathrm{Na}$ análise funcional, o cientista do comportamento busca identificar relações de dependência entre diferentes eventos que ocorrem ao mesmo tempo e em uma dada ordem. Supõe-se que tais relações permitem identificar as causas mais prováveis dos comportamentos, permitindo sua descrição, explicação, predição e controle (Ulian, 2007). Segundo Skinner (1953/1965), o analista do comportamento não usa os termos causa e efeito com os mesmos significados que no senso comum e em outras áreas do conhecimento. Para o autor, falar em causa e efeito significa dizer que certos eventos ocorrem em certa ordem, sem ênfase em dizer como isso acontece. Assim, uma explicação do comportamento requer análise funcional, isto é, requer a identificação das variáveis das quais o comportamento é função.

O conceito de análise funcional é, portanto, atrelado à concepção de comportamento operante, representada pela contingência de, pelo menos, três termos. Nesta, a resposta do indivíduo é considerada como variável dependente e os estímulos antecedentes e consequentes como variáveis independentes (Neno, 2003; Skinner, 1953/1965; Ulian, 2007).

Ao observar relações regulares entre indivíduo e ambiente, ou entre respostas e estímulos, a análise funcional parte da premissa da multideterminação do fenômeno comportamental, bem como do reconhecimento da variabilidade de combinações possíveis entre as determinações múltiplas que afetam o indivíduo, entendendo a noção de causalidade do termo "relação funcional" como probabilística (Neno, 2003; Skinner, 1953/1965; Ulian, 2007). O externalismo vigora como recorte de análise e o modelo de seleção por consequências como mo- delo causal, de tal forma que o comportamento é entendido além de suas topografias (Neno, 2003; Sturmey et al., 2007).

Embora o conceito de análise funcional seja central na pesquisa e na prática do analista do comportamento, existem inconsistências na formulação do termo na literatura analítico-comportamental. Tanto em nível nacional quanto internacional, há diferentes termos com atribuições iguais ou semelhantes, como avaliação funcional e análise de contingências (Andery, Micheletto \& Sério, 2001; Haynes \& O’Brien, 1990; Neno, 2003; Virués-Ortega \& Haynes, 2005). Além disso, prevalecem posições distintas em relação aos objetivos, métodos de implementação e componentes relevantes na formulação da análise funcional. Nesse sentido, Neno (2003) aponta que a ausência de consenso sobre o que de fato significa a análise funcional aparece de modo mais evidente na literatura de aplicação clínica.

Por exemplo, Haynes e O’Brien (1990) assumem que uma variável causal é aquela que, quando modificada, produz mudanças em ao menos um dos parâmetros da variável dependente (como probabilidade, magnitude ou taxa da resposta à qual se relaciona). Assim, no contexto de terapia, eles definem análise funcional como a identificação de relações funcionais relevantes, controláveis e causais, que se aplicam a comportamentos específicos de um dado indivíduo. Porém, como poderia uma relação funcional ter, simultaneamente, variáveis que sejam relevantes, controláveis e causais? Os autores apontam que seria difícil atingir por completo essa definição e, com isso, se aproximam de Skinner, concluindo que análises funcionais nem sempre explicam totalmente o comportamento, apesar de o cientista buscar esse objetivo "à exaustão". Ao contrário, elas devem ajudar na identificação de variáveis causais importantes, isto é, que possam ser manipuladas. Portanto, a análise funcional, para os autores, deve enfatizar o nível mais relevante da relação funcional em questão, tendo em vista a aplicação desejada.

A definição proposta por Haynes e O'Brien (1990) também é adotada por Virués-Ortega e Haynes (2005). No entanto, tais autores consideram que relações funcionais podem não ser causais, variar em força (i.e., no grau de influência que exer- 
cem sobre o comportamento), ser dinâmicas (i.e., com mudanças em variáveis causais, o mecanismo de mediação muda ou outras variáveis passam a afetar o comportamento, que, consequentemente, fica alterado), ter distintos níveis de especificidade (i.e., podem ser molares, avaliando os impactos que o problema está trazendo no funcionamento global do cliente, ou moleculares, analisando o efeito momentâneo e específico de variáveis ambientais sobre determinada resposta) e, ainda, ser uni ou bidirecionais (em relações bidirecionais, duas variáveis se afetam entre si, uma a outra).

A definição adotada por Haynes e O'Brien (1990) e Virués-Ortega e Haynes (2005), contudo, corresponde a um tipo de análise funcional em que as variáveis envolvidas podem ser classificadas como causais ou correlacionais, modificáveis ou não e importantes em força. Neste caso, entende-se que a análise funcional seria probabilística e não puramente determinista. Além disso, as relações envolvidas seriam não exclusivas, pois uma relação entre duas variáveis não exclui relações entre essas e outras variáveis (Ulian, 2007).

Outro tipo de análise funcional, usualmente empregada por terapeutas comportamentais, trata-se de um método de formulação de caso em que são buscadas as variáveis de controle do comportamento a partir de hipóteses (ou seja, interpretações) das possíveis variáveis que mantêm os comportamentos, tais como nos trabalhos de Leonardi, Borges e Cassas (2012) e de Yoman (2008). Em especial, Yoman enfatiza o modo como os dados da análise são coletados e descreve três subtipos de análise funcional como método de formulação de caso. No primeiro tipo - análises funcionais de avaliações baseadas em informantes - os dados são coletados a partir de questionários, entrevistas ou fichas de automonitoramento com o cliente ou com indivíduos próximos a ele. Nas avaliações observacionais, o segundo tipo, o profissional coleta dados a partir de observações diretas do comportamento-alvo, de modo que o teste da hipótese inicial é feito pré e pós intervenção. Por fim, o terceiro tipo diz respeito a análises funcionais experimentais (o padrão-ouro), nas quais o teste de hipóteses é feito com base em um delineamento experimental de caso único, seja pelo método de reversão ou de linha de base múltipla.
Sturmey, War-Hormer, Marroquin e Doran (2007) afirmam que a análise funcional requer que o terapeuta tenha controle sobre o comportamento em questão e a habilidade de fazê-lo ocorrer ou cessar. Assim sendo, os autores argumentam que as intervenções em casos clínicos não devem ser formuladas a partir de autorrelatos dos clientes sobre os seus próprios comportamentos, e sim derivar da identificação dos antecedentes e consequentes relacionados a eles por meio do método experimental. No entanto, a exigência do uso exclusivo do método experimental na realização de análises funcionais é controversa na literatura. Nessa direção, Ulian (2007) argumenta que a identificação da função de um comportamento por meio de testes experimentais nem sempre é passível de aplicação na clínica.

A multiplicidade de caracterizações e usos da análise funcional é explicitada na variedade de termos com o mesmo significado na literatura (Haynes \& O'Brien, 1990; Neno, 2003; Sturmey et al., 2007; Ulian, 2007). Em diversos casos, análise funcional corresponde a uma fase de "diagnóstico", anterior à intervenção, na qual são identificadas as variáveis das quais o comportamento é função. Diferenciase, então, análise funcional de avaliação comportamental. Neste caso, a avaliação comportamental é entendida como uma análise completa, maior do que a análise funcional, pois inclui a identificação do comportamento-problema com suas variáveis mantenedoras, proposta de intervenção, sua execução e monitoramento dos resultados obtidos. Por outro lado, muitas vezes o termo análise funcional denota o processo que envolve desde o levantamento de variáveis relevantes até o estabelecimento de intervenções, correspondendo, portanto, a um diagnóstico constante do comportamento. Esse uso do termo análise funcional é, com frequência, adotado na clínica. Nesse caso, o resultado da intervenção serve como medida de validade das variáveis de controle hipotetizadas pelo terapeuta.

Além da problemática relacionada à multiplicidade de caracterizações e usos da análise funcional, vale destacar que essa ferramenta parece ser subutilizada dentro da própria terapia comportamental. Por exemplo, Haynes e O'Brien (1990) mostram que apenas 20\% de 156 intervenções comportamentais foram baseadas em dados de análise funcional pré- 
-intervenção. De modo semelhante, Virués-Ortega e Haynes (2005), 15 anos depois, revelam o baixo número de pesquisas experimentais com avaliação de caso clínico a partir de análise funcional.

No Brasil, a análise funcional vem sendo utilizada em diferentes modelos de terapia pautados na Análise do Comportamento. Costa (2011) articula reflexões a respeito de distintas denominações dadas à terapia comportamental no Brasil na última década e avalia como a análise funcional é caracterizada e desenvolvida em cada uma delas. Assim, ao verificar as semelhanças e diferenças entre terapia analítico-comportamental (TAC), terapia por contingências de reforçamento (TCR), terapia molar e de autoconhecimento (TMA) e psicoterapia comportamental pragmática ( $\mathrm{PCP})$, a autora identifica que, em todas elas, a análise funcional é colocada como ferramenta fundamental ao longo de todo o processo terapêutico.

Recentemente, Leonardi (2016), em uma revisão sistemática sobre a produção de evidências empíricas de eficácia da terapia comportamental de origem brasileira (ou seja, TAC, TCR, etc.), revelou que a análise funcional foi empregada em apenas 29.5\% dos 44 casos analisados. Em outras palavras, menos de um terço de todas as publicações apresentaram hipóteses funcionais relacionadas ao comportamento-alvo da intervenção, o que parece distanciar o trabalho clínico das características essenciais da prática do analista do comportamento. Entretanto, conforme o autor explicita, não foi realizado em seu trabalho um exame minucioso das características das análises funcionais nos 29.5\% dos casos, sugerindo que um novo estudo deveria se debruçar sobre isso.

Em suma, tendo em vista (1) a relevância da análise funcional para a prática do terapeuta analítico-comportamental e (2) os resultados de Leonardi (2016), fica evidente a necessidade de avaliar o uso da análise funcional nos estudos brasileiros. Em vista disso, o presente trabalho teve por objetivo investigar as características das análises funcionais dos casos revisados por Leonardi (2016), a fim de oferecer um panorama do uso da análise funcional na literatura brasileira de terapia analítico comportamental.

\section{Método}

O procedimento de seleção de dados e sistematização da amostra da literatura envolveu três etapas, listadas abaixo e descritas em seguida:

- Fase 1: Procedimento de coleta de dados;

- Fase 2: Leitura dos trabalhos selecionados e sistematização dos dados;

- Fase 3: Análise dos dados.

\section{Fase 1: Procedimento de coleta de dados}

Tendo em vista o objetivo do presente estudo, o procedimento de coleta dos trabalhos para compor a amostra foi desenvolvido a partir de Leonardi (2016). Em seu trabalho, o autor teve como objetivo avaliar criticamente a produção de evidências empíricas acerca da eficácia da TAC e da FAP na literatura nacional e internacional e, a fim de abarcar o maior número possível de estudos empíricos, publicados ou não, conduziu a busca em onze bases de dados globais e três bases de dados específicas de análise do comportamento. Dos 72 casos revisados pelo autor, 31 eram do Brasil e pontuaram "sim" na categoria "análise de contingências", isto é, esses foram os trabalhos brasileiros que apresentaram alguma hipótese funcional relacionada ao alvo da intervenção.

Inicialmente, a proposta do presente trabalho era categorizar todas as análises funcionais desses 31 trabalhos. No entanto, uma análise preliminar dos dados revelou que isso seria inviável porque os textos não diferenciam o que propriamente é a análise funcional do que é uma observação do autor sobre o caso sem que tenha o compromisso de estabelecer relações entre variáveis. A fim de assegurar o rigor científico e a validade dos dados obtidos, foram excluídos da amostra os textos que apenas descreveram as contingências analisadas, sendo incluídos apenas os trabalhos que as desenharam ou as organizaram em forma de tabela. Além disso, o trabalho de Borges (2010) foi excluído porque a figura desenhada pelo autor apresentava símbolos (e.g., "Sa" como "antecedente", "Ra1" como "resposta" e "Ca1" como "consequência") sem descrição dos elementos. Dessa forma, 11 trabalhos compuseram a amostra do presente estudo, listados no Apêndice 1, totalizando 13 casos clínicos analisados. 
Fase 2: Leitura dos trabalhos selecionados e sistematização dos dados

Após a seleção dos trabalhos, foi realizada a leitura integral dos 13 casos com vistas a assegurar maior compreensão dos dados e precisão no registro. A ordem de leitura empregada seguiu a sequência alfabética das referências. Um banco de dados foi construído em uma planilha do Microsoft Excel, no qual cada linha correspondeu a cada contingência desenhada em cada caso da amostra. Por sua vez, cada coluna correspondeu a uma das categorias de coleta ou de análise. O que, nos textos, delimitava o conteúdo de cada contingência era a própria divisão gráfica dos desenhos, feitas pelos autores da amostra. No trabalho de Rocha (2012), o único em que não houve divisão gráfica das contingências, considerou-se uma unidade de contingência tendo como base a resposta. Para trabalhos com mais de um caso, sua referência foi repetida na planilha, respeitando a organização de uma contingência desenhada por linha do Microsoft Excel. Para casos com mais de uma contingência desenhada, manteve-se esse padrão. Enfim, se no trabalho estava descrita a categoria de coleta em questão, o trecho correspondente no texto foi copiado para a planilha, possibilitando a computação dos dados. Por exemplo, se o autor descreveu antecedentes, esses foram copiados para coluna correspondente na tabela do Excel. Os demais elementos desenhados na contingência que se relacionavam a esse antecedente, foram copiados na mesma linha, nas respectivas colunas correspondentes. Caso o autor não tenha descrito o dado relacionado a quaisquer das categorias analisadas, foi escrito "NI" (não informa) na célula correspondente. Uma vez preenchidas todas as informações referentes às categorias de coleta da contingência em questão, foram preenchidas as categorias de análise de análise conforme descrito a seguir.

O banco de dados foi composto a partir das categorias e variáveis elaboradas pelos autores e descritas abaixo. Essa maneira de organizar os dados teve como base a interpretação destes pesquisadores acerca da descrição apresentada nos textos analisados, exclusivamente. Isso se deve ao fato de que, conforme alertou Leonardi (2016), não existe uma metodologia de pesquisa sistematizada e compartilhada por um grande número de pesquisadores que oriente a confecção deste tipo de trabalho. Isso posto, fica claro que o procedimento empregado no estudo é idiossincrático, inviabilizando replicação direta.

A fim de reduzir vieses na produção do banco de dados da pesquisa e aumentar a relevância dos resultados obtidos, as informações foram coletadas e analisadas por duas pesquisadoras (a primeira e a segunda autoras deste artigo). Esse processo ocorreu a partir de um estudo piloto, no qual foram lidos e categorizados os dois primeiros textos do Apêndice 1. Na sequência, foram discutidos os dados obtidos e alteradas algumas categorias e variáveis, tornando as descrições mais precisas. Por fim, os demais textos da amostra foram lidos e categorizados. Dessa forma, o objetivo em ter uma segunda pesquisadora durante a coleta foi suscitar, discutir e solucionar dúvidas e divergências, sem intenção de promover uma porcentagem de concordância. Enfim, foram lidas, analisadas e sistematizadas as informações dos 13 casos selecionados de acordo com os critérios anteriormente apresentados, a partir das variáveis e categorias de coleta descritas a seguir:

\section{Categorias de coleta referentes aos dados gerais}

\section{Referência}

\section{Ano}

3. Tipo da pesquisa: relato de caso, experimento de caso único ou pesquisa de grupo. Nos casos de experimento de caso único foi registrado o delineamento adotado no trabalho (e.g. AB; $\mathrm{ABAB}$ - reversão, linha de base múltipla, etc). Essa categoria seguiu os dados já computados por Leonardi (2016).

4. Fonte de dados: análise funcional baseada em informantes (INF) - quando a coleta de dados ocorreu a partir de terceiros à terapia; análise funcional a partir de material estruturado (EST), como questionários, escalas ou folhas de automonitoramento que abordam o cliente ou indivíduos próximos do mesmo; análise funcional baseada na interação terapeuta-cliente sem estrutura pré-definida (NEST) - quando a coleta dados ocorreu a partir de diálogos e observações do comportamento relevante, seja ele 
verbal ou não. Nos casos de EST, foi registrado o material de literatura empregado pelo pesquisador (e.g., Questionário de História Vital, de Lazarus, 1980).

5. Termo empregado: registro do termo exato empregado pelo autor (e.g., análise de contingência, análise funcional, avaliação funcional, etc).

6. Autor de referência para a definição adotada de análise funcional: transcrição da (s) referência (s) citada (s) e registro se o (s) autor (es) estabeleceu (estabeleceram) relações matemáticas na análise funcional (MAT).

\section{Categorias de coleta referentes às contingências analisadas}

1. Número de termos: quantidade de termos que compuseram cada contingência analisada.

\section{Antecedente:}

- Nome dado ao "antecedente": foram registrados os termos que os autores usaram para nomear o que foi desenhado como "antecedente". A saber: antecedente (s), antecedentes históricos, evento antecedente, SD1, SD2, Sds e situação antecedente. Este critério foi adotado também para o nome da "resposta", da "consequência" e do "efeito emocional".

- Descrição do "antecedente": foram registradas as palavras empregadas pelo autor para descrever o "antecedente", copiando-as do texto original. Este critério foi adotado também para a descrição da "resposta", da "consequência" e do "efeito emocional".

\section{Resposta:}

- Nome dado à "resposta": comportamento, respostas, encobertos / públicos, respostas e sentimentos, C1, C2, comportamento de Isadora.

- Descrição da "resposta".

\section{Consequência:}

- Nome dado à "consequência": consequência(s); consequências ambientais e efeitos emocionais; consequentes.

- Descrição da "consequência”.
5. Efeito emocional: emoções / sentimentos / sensações apontadas pelo (s) autor (es) separadamente dos antecedentes, respostas e consequências.

- Nome do "efeito emocional": frequência e efeitos.

- Descrição do "efeito emocional".

\section{Fase 3: Análise dos dados}

A partir da coleta e sistematização dos dados, foram elaboradas categorias de análise. Para todas as categorias, foi pontuado "NA" ("Não se Aplica") nos casos em que a categoria avaliada não fosse aplicável ao trabalho ou à contingência em questão e "NID" quando "não foi possível identificar" o dado correspondente à categoria em questão. Conforme dito anteriormente, pontuou-se "NI" ("não informa") para todos os casos em que o autor não informou o dado referente à categoria avaliada. A seguir estão dispostas as categorias de análise empregadas no presente trabalho, de acordo com o termo da contingência ao qual se referem:

\section{Antecedente:}

- Soma de eventos desenhados pelos autores como "antecedente" de cada contingência: nesse caso, foi considerada uma unidade quando o autor separou por sinal gráfico uma sequência de palavras (e.g., em "Estar em casa; Cobranças da esposa e dos filhos", foi pontuado "2" nessa categoria). Esse critério foi adotado para a mesma categoria de análise referente à "resposta" e à "consequência".

- Tipo de "antecedente": estímulo antecedente (SANT), história de vida (HV), resposta (R) ou não-resposta (NR). Nesse caso, foi considerado como HV quando o autor descreveu elementos históricos e não estímulos antecedentes para a resposta sob análise (e.g., no caso em que "abusada sexualmente, aos 4 anos de idade, por dois primos maiores de idade" foi desenhado como "antecedente", e 'Pensa: "eu deixei que eles fizessem aquilo, pois sentia uma sensação boa e porque eles me levam para andar a cavalo"' foi desenhado como "resposta"). Além disso, nos casos de relatos verbais como "antecedentes", considerou-se SANT quando o autor colocou o relato do cliente na sessão que informava sobre o estímulo antecedente (e.g., em "Minha mãe 
brigou comigo, pois não fiz todas as tarefas designadas por ela", foi computado "SANT"). Isso ocorreu também quando o autor deixou explícito se tratar se uma regra, autorregra ou pensamento com função de estímulo antecedente (e.g., "Ter que lavar as louças de seu lanche antes de os pais chegarem em casa" ou "Ordem do namorado para que volte à casa das irmãs" ou "Não pode frequentar a casa de amigas"). Nos demais casos, foi pontuado R (e.g., "Fiz todas as tarefas que minha mãe pediu" ou "Deixei o arroz para minha irmã fazer"). Considerou-se NR quando o autor desenhou uma não-resposta como "antecedente", como em: "Não recusa pedidos abusivos".

- Soma dos SANT: número total de SANT pontuados anteriormente.

- Condição do SANT: social não-verbal (SOC), físico (FIS) ou verbal (V). Nesse caso, foi pontuado SOC para os estímulos que eram sociais e não-verbais, pois todo estímulo verbal é social. Além disso, pontuou-se FÍS para estímulos inanimados que evoquem uma resposta.

- Imediaticidade temporal: pontuou-se "SIM" quando o (s) SANT da contingência fosse (m) temporalmente imediato $(\mathrm{s})$ à $(\mathrm{s})$ resposta $(\mathrm{s})$. Caso contrário, pontuou-se "NÃO".

\section{Resposta:}

- Soma de eventos desenhados pelo autor como "resposta" de cada contingência.

- Tipo de "resposta": o que o autor desenhou como "resposta" da contingência corresponde a um comportamento respondente $(\mathrm{R})$, comportamento operante $(\mathrm{O})$, ambos $(\mathrm{R}+\mathrm{O})$, emoção/sentimento/sensação (E), consequência (C) ou não-resposta (NR). Nesse caso, pontuou-se $\mathrm{R}+\mathrm{O}$ nos casos em que, apesar de o autor não ter apresentado uma contingência respondente, um procedimento respondente seria efetivo enquanto intervenção (e.g., "disfunção erétil"). - Soma das respostas: soma do que foi pontuado como R, O ou R+O.

- Foco da resposta: a resposta da contingência (i.e., o que foi pontuado como $\mathrm{R}, \mathrm{O}$ ou $\mathrm{R}+\mathrm{O}$ ) corresponde a um comportamento problema (PROB) ou comportamento solução (SOL).
Considerou-se PROB os comportamentos envolvidos na demanda do cliente, que deveriam ter a frequência reduzida com a terapia. Por outro lado, SOL correspondeu aos comportamentos de melhora, que a intervenção tinha como objetivo aumentar.

- Repertório verbal: a resposta da contingência (i.e., o que foi pontuado como $\mathrm{R}, \mathrm{O}$ ou $\mathrm{R}+\mathrm{O}$ ) se refere a uma interação verbal (RV) ou não-verbal (RNV). Neste caso, foi avaliada a propriedade essencial da resposta para diferenciar entre RV e RNV. Por exemplo, em "ligar para o namorado com frequência" ou em "ameaça ir embora" foi computado RV, ainda que não fique claro se o autor se referia ao ato de digitar números ou a deslocar-se para a saída. Por outro lado, "chora" foi considerada RNV.

- Operacionalização: a resposta da contingência é operacionalizada (COPE) (e.g., o autor descreve "jogar-se no chão" ao invés de "birra") ou não-operacionalizada (NCOPE).

\section{Consequência:}

- Soma de eventos desenhados pelo autor como "consequência" de cada contingência.

- Tipo de "consequência": o que o autor desenhou como "consequência" da contingência corresponde a um estímulo punidor negativo (SAV-), estímulo punidor positivo $(\mathrm{SAV}+)$, estímulo reforçador negativo (SR-), estímulo reforçador positivo (SR+), resposta (R), não-resposta (NR), emoção/sentimento/sensação (E), extinção (EXT) ou função (F). Neste caso, foi considerado EXT ou $\mathrm{F}$ quando o autor não apontou um estímulo como "consequência", mas sim um processo (e.g., em "fuga/esquiva" ou em "reforçamento negativo" pontuou-se F). Nas contingências em que o autor menciona a função do comportamento mas adiciona o evento (seja estímulo, seja resposta), foi categorizado o evento em questão. Por exemplo, em "fuga/esquiva de fazer a tarefa" foi pontuado $\mathrm{R}$ pois a "consequência" descrita foi evitar uma resposta, ou seja, é uma resposta. Já em "remoção de situações sociais", foi pontuado SR-.

- Soma das consequências: soma do que foi pontuado como SAV+, SAV-, SR+, SR- ou EXT. 
- Imediaticidade temporal: pontuou-se "SIM" quando a consequência da contingência fosse temporalmente imediata à (s) "resposta (s)". Caso contrário, pontuou-se "NÃO”. Esta categoria se aplicou somente aos casos anteriormente pontuados como SAV+, SAV-, SR+, SRou EXT.

- Relação funcional: pontuou-se "SIM" quando a consequência da contingência fosse produzida pela (s) "resposta (s)", estabelecendo relação funcional. Caso contrário, pontuou-se "NÃO". Essa categoria também se aplicou somente aos casos anteriormente pontuados como SAV+, SAV-, SR+, SR- ou EXT.

\section{Resultados}

Toda a amostra foi composta por relatos de caso e todos os trabalhos partiram de informações coletadas sem estrutura definida, isto é, a partir de observações do terapeuta e do relato do cliente na sessão. Nesse contexto, sobressaem-se os sete casos em que, de modo adicional, empregaram materiais estruturados na coleta de informações (são eles: Batista, 2008; Bueno, 2009; Elias, 2005; Moriyama, 2007; Rocha, 2012), sendo que em cinco deles foi utilizada folha de registro / monitoramento dos comportamentos do cliente. Vale destacar que Bueno (2009) foi o único caso que fez uso de relatos de informantes terceiros à terapia.

Somente em dois dos 13 casos da amostra foram identificados os autores de referência para definir análise funcional. Bueno (2009) cita Skinner (1953/2000) e Moriyama (2007) faz um resgate de autores (citando, entre outros, Neno, 2003 e Skinner, 1953/1965). Somado a isso, nove autores nomearam a prática de "análise funcional", enquanto quatro não atribuíram um nome para a sua atuação. Não houve casos da amostra em que os autores afirmaram fazer "análise de contingências" e nem casos em que foram estabelecidas relações matemáticas.

Das 72 contingências analisadas nesta pesquisa, cinco apresentaram dois termos, 12 apresentaram quatro termos e a grande maioria, 55, corresponderam a contingências de três termos. Vale destacar, ainda, que em seis das 12 contingências com quatro termos foram colocados emoções, sentimen- tos e sensações separadamente dos "antecedentes", "respostas" e "consequências". Em quatro dessas 12 contingências, o autor separou respostas encobertas de públicas e, em uma, o autor inseriu separadamente a história relacionada à contingência.

Houve discrepância entre a quantidade de eventos que os autores desenharam como "antecedentes" e o que foi avaliado na análise como sendo estímulo antecedente (SANT). Conforme mostra a Tabela 1, por exemplo, em 53 das 72 contingências avaliadas os autores desenharam um evento no campo "antecedente" da contingência $(\mathrm{N}=1)$. Porém, na análise dos dados, verificou-se que em apenas 38 das 72 contingências foi desenhado um estímulo antecedente (SANT). Além disso, em dez das contingências desenhadas na amostra, ainda que os autores tivessem afirmado ter descrito "antecedentes", não foram computados estímulos antecedentes (SANT) na análise dos dados $(\mathrm{N}=0)$.

\section{Tabela 1. Número de Contingências em que Foram Desenhados N Eventos Como "Antecedente" e Número de Contingências em que N Eventos Foram Pontuados na Análise como SANT}

\begin{tabular}{ccc}
\hline & $\begin{array}{c}\text { Número de contingências } \\
\text { em que foram desenhados } \\
\text { N eventos como } \\
\text { "antecedente" }\end{array}$ & $\begin{array}{c}\text { Número de } \\
\text { contingências em } \\
\text { que N eventos foram } \\
\text { pontuados na análise } \\
\text { como SANT }\end{array}$ \\
\hline $\mathrm{N}=0$ & 0 & 10 \\
$\mathrm{~N}=1$ & 53 & 38 \\
$\mathrm{~N}=2$ & 5 & 11 \\
$\mathrm{~N}=3$ & 3 & 2 \\
$\mathrm{~N}=4$ & 1 & 0 \\
$\mathrm{~N}=5$ & 0 & 1 \\
TOTAL & 62 & 62 \\
\hline
\end{tabular}

Tais dados podem ser melhor compreendidos quando avaliado o tipo de "antecedente", pois, do total de 92 eventos desenhados, 11 foram analisados como sendo história de vida (HV), nove como resposta (R) e um como não-resposta (NR). Ainda assim, fica claro que, para grande parte das contingências da amostra (71 dos 92 eventos), foi avaliado que os autores colocaram estímulos antecedentes (SANT) no que foi desenhado como "antecedente", correspondendo a $65,7 \%$ do total de eventos. Em 
15 eventos foi pontuado NA e em um, NID. Tais informações estão organizadas em forma de imagem na Figura 1.

Ainda no que se refere ao antecedente, em 65 das 71 vezes em que foi pontuado SANT durante a análise (91,55\%), o estímulo foi temporalmente imediato à resposta correspondente. Apenas em duas vezes não houve imediaticidade temporal de acordo com a análise e em quatro vezes não foi possível identificar a imediaticidade. Além disso, 44 dos 71 SANTs contabilizados foram avaliados como sociais (S) e 18 como verbais (V). Somente sete SANTs foram considerados físicos $(\mathrm{F})$ e dois pontuaram NID.

Em relação ao campo resposta da contingência, o número de contingências em que os autores desenharam uma "resposta" $(\mathrm{N}=1)$ nesse campo da contingência e o número de contingências em que foi pontuada uma $(\mathrm{N}=1)$ resposta $(\mathrm{R})$ na análise foi semelhante: respectivamente 50 e 49 das 72 contingências. Essa concordância pode ser observada de modo geral na Tabela 2, o que sugere que os autores da amostra estão identificando ações na hora de desenhar as "respostas" das análises de contingências.

Tabela 2. Número de Contingências em que Foram Desenhados N Eventos Como "Resposta" e Número de Contingências em que $\mathrm{N}$ Eventos Foram Pontuados na Análise como Resposta (R, $\mathrm{O}$ ou $\mathrm{R}+\mathrm{O}$ )

\begin{tabular}{ccc}
\hline & $\begin{array}{l}\text { Número de } \\
\text { contingências em que } \\
\text { foram desenhados } \\
\text { N eventos como } \\
\text { "resposta" }\end{array}$ & $\begin{array}{l}\text { Número de } \\
\text { contingências em } \\
\text { que N eventos } \\
\text { foram pontuados na } \\
\text { análise como sendo } \\
\text { resposta }\end{array}$ \\
\hline N=0 & 0 & 2 \\
N=1 & 50 & 49 \\
N=2 & 7 & 10 \\
N=3 & 10 & 6 \\
N=4 & 4 & 3 \\
N=5 & 0 & 0 \\
N=6 & 0 & 1 \\
NA & 1 & 1 \\
TOTAL & 72 & 72 \\
\hline
\end{tabular}

Em relação ao tipo da "resposta", tal como mostra a Figura 2, destaca-se a maioria de comportamentos operantes, totalizando 101 eventos $(79,5 \%)$. Avaliou-se que respostas exclusivamente respondentes não foram desenhadas nenhuma vez e, em três ocasiões, foram pontuadas respostas operantes e respondentes $(\mathrm{R}+\mathrm{O})$. Além disso, emoções/sentimentos/sensações (E) foram pontuados por 15 vezes desenhados como "resposta" da contingência e uma "não-resposta" (NR) foi avaliada em quatro casos, a saber: "não há cobranças com o namorado" (Ferreira e Farias, 2009), "não mexe nas coisas dele, procurando indícios de traições" (Ferreira e Farias, 2009), "não confia nas pessoas" (Rocha, 2012) e "não fala sobre a vida pessoal com os pais" (Rocha, 2012). Em um único caso avaliou-se que uma consequência (C) estava desenhada na "resposta" da contingência; em três casos foi pontuado NA e, em um caso, NID.

Outros dados se referem ao que foi pontuado como R, O, R+O ou NID em termos de foco da resposta, operacionalização e repertório verbal. Das 105 ocorrências analisadas, 88 focaram no comportamento-problema (PROB), 14 no comportamento-solução (SOL) e três não foram identificadas (NID). Nesse total (105), foi semelhante à soma das respostas operacionalizadas (COPE) e não-operacionalizadas (NCOPE), respectivamente 55 e 50. Ademais, 54 respostas foram pontuadas como resposta verbal (RV), 26 como resposta não-verbal (RNV) e 25 como NID.

Em relação ao campo consequência da contingência, em 46 das 72 contingências os autores desenharam uma "consequência" $(\mathrm{N}=1)$. Porém, na análise do presente trabalho, avaliou-se que foram empregados estímulos consequentes à resposta (i.e., SAV+, SAV-, SR+, SR- ou EXT) em 28 contingências, enquanto que por 12 vezes não foram identificados, conforme ilustra a Tabela 3 .

Essa característica pode ser mais bem compreendida quando avaliado o tipo de "consequência" de acordo com as categorias de análise. Conforme ilustrado na Figura 3, a "consequência" mais frequente foi o estímulo reforçador negativo (SR-), que apareceu 43 vezes. Em seguida, com 37 ocorrências, o estímulo punidor positivo (SAV+). Em terceiro lugar, com 26 ocorrências, foram pontuadas respostas (R) desenhadas no campo "consequência" da 
contingência, próximo ao número de emoções/sentimentos/sensações (24 ocorrências) e de estímulo reforçador positivo (23 ocorrências). Sete vezes foi pontuado NA, quatro vezes extinção (EXT), três vezes não-resposta (NR) e três vezes função $(\mathrm{F})$. Por fim, o menos pontuado foi o estímulo punidor negativo (SAV-), totalizando duas ocorrências.

Tabela 3. Número de Contingências em que Foram Desenhados N Eventos Como "Consequência" e Número de Contingências em que $\mathrm{N}$ Eventos Foram Pontuados na Análise Como Sendo Consequência (SAV+, SAV-, SR+, SR- ou EXT)

\begin{tabular}{ccc}
\hline & $\begin{array}{l}\text { Número de } \\
\text { contingências } \\
\text { em que foram } \\
\text { desenhados N } \\
\text { eventos como } \\
\text { "consequência" }\end{array}$ & $\begin{array}{l}\text { Número de contingências } \\
\text { em que N eventos } \\
\text { foram pontuados na } \\
\text { análise como sendo } \\
\text { consequência (SAV+, } \\
\text { SAV-, SR+, SR- ou EXT) }\end{array}$ \\
\hline N=0 & 3 & 12 \\
N=1 & 46 & 28 \\
N=2 & 8 & 15 \\
N=3 & 6 & 7 \\
N=4 & 3 & 3 \\
N=5 & 3 & 2 \\
N=6 & 1 & 0 \\
N=7 & 1 & 0 \\
N=8 & 0 & 1 \\
NA & 1 & 42 \\
TOTAL & 72 & \\
\hline
\end{tabular}

Quanto à relação resposta-consequência, em 79 das 108 vezes (ou seja, 73,1\%) foi pontuado que houve ("SIM") imediaticidade temporal da consequência (i.e., do que foi pontuado como SR-, SR+, SAV-, SAV+ ou EXT), em oposição a 24 casos em que se analisou que a consequência elencada não foi imediata à emissão da resposta ("NÃO"). Por exemplo, para a resposta "tentativa de suicídio" a consequência "ele ri dela" (Ferreira e De-Farias, 2009) foi pontuada como "SIM". Por sua vez, para a contigência em que foi desenhado como "antecedente" "traições do namorado", como "resposta" "aceita sempre as desculpas dele" e como "consequência" "novas traições" (Ferreira; De-Farias, 2009) foi pontuado "NÃO". Outro dado é que na expressiva maioria das vezes (em 93 dos 108, ou seja, 88,5\%), considerou-se que os autores elencaram estímulos consequentes que estabeleciam relação funcional com a resposta. Somente em 15 vezes não foi identificada relação funcional.

Por fim, para facilitar a compreensão do leitor do conjunto dos dados obtidos, as Figuras 1, 2 e 3 apresentam os tipos do que os autores descreveram, respectivamente, nos campos antecedente, resposta e consequência das contingências.

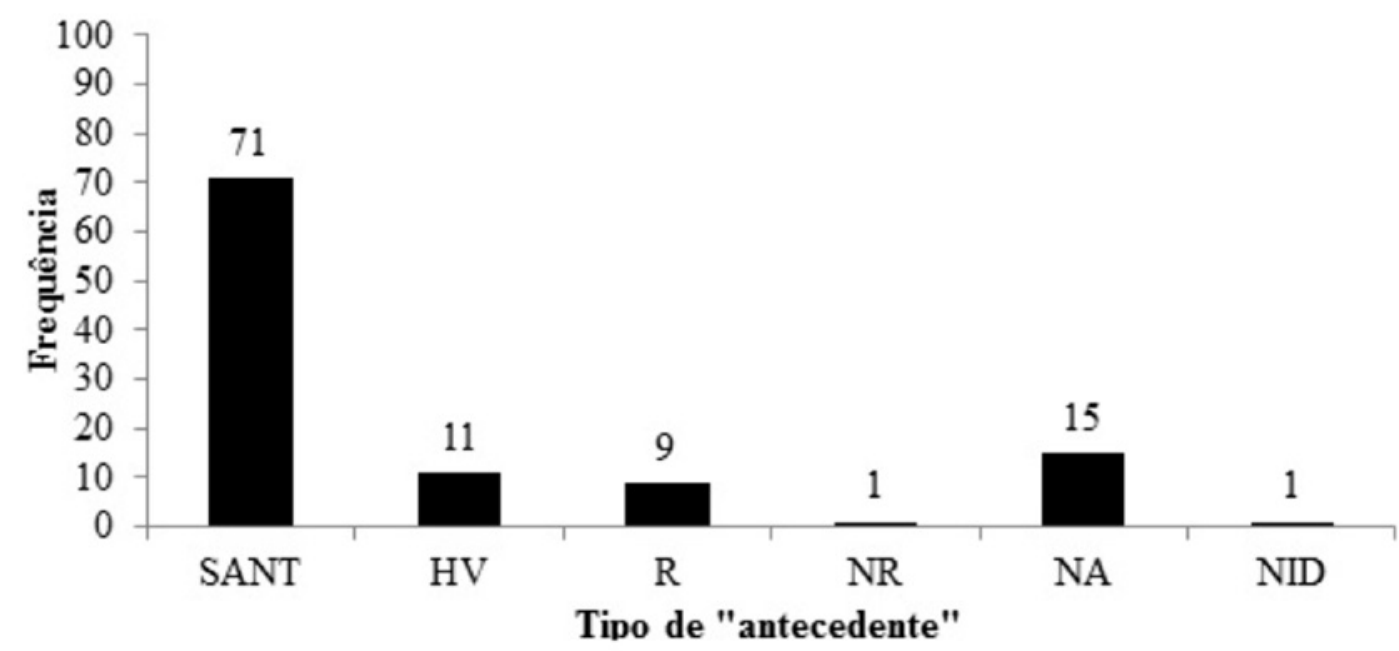

Figura 1. Frequência dos tipos de "antecedente"analisados, sendo eles: estímulo antecedende (SANT), história de vida (HV), resposta (R), não resposta (NR), não se aplica (NA) ou não identificada (NID). 


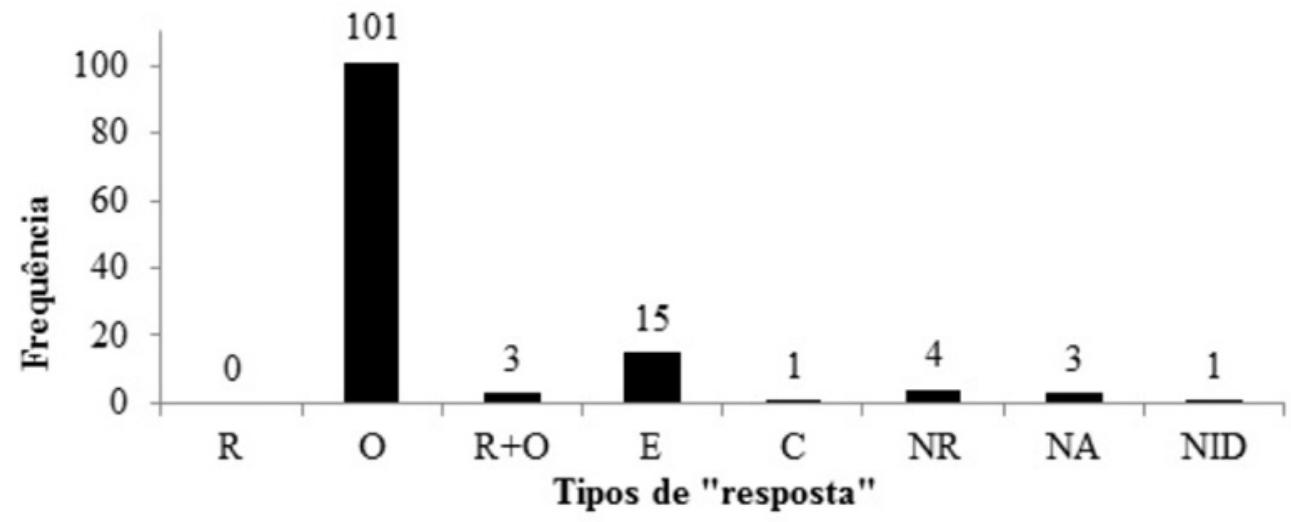

Figura 2. Frequência dos tipos de "resposta" analisados, sendo eles: respondente (R), operante (O), respondente + operante $(\mathrm{R}+\mathrm{O})$, emoção/sentimento/sensação $(E)$, consequência (C), "não-resposta" (NR), não se aplica (NA) ou não identificada (NID).

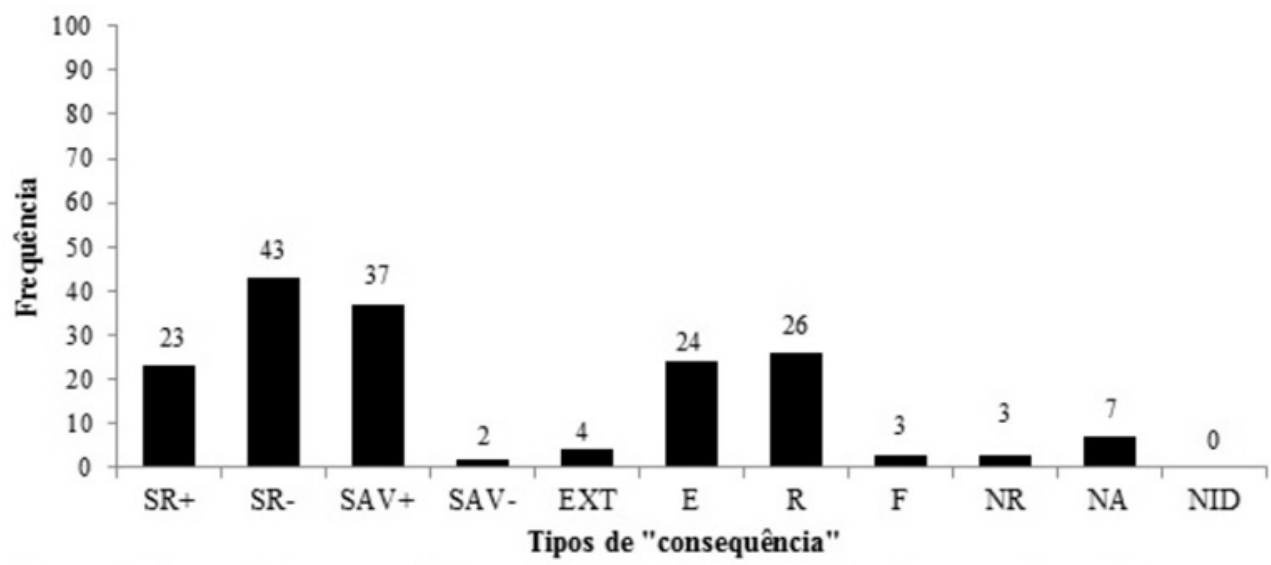

Figura 3. Frequência dos tipos de "consequência"analisados, sendo eles: estímulo punidor negativo (SAV-), estímulo punidor positivo (SAV+), estímulo reforçador negativo (SR-), estímulo reforçador positivo (SR+), resposta $(R)$, não-resposta $(N R)$, emoção/sentimento/sensação $(E)$, extinção $(E X T)$, função $(F)$, não se aplica (NA) ou não identificada (NID).

\section{Discussão}

Os resultados encontrados ilustram as principais características do uso da análise funcional em estudos brasileiros que descrevem intervenções voltadas a clientes típicos atendidos no ambiente de consultório.

A contingência de três termos - antecedente, resposta e consequência - vem sendo a mais adotada, tal como sugerido por Skinner (1953/1965) e reiterado por diversos autores (como Leonardi et al., 2012; Neno, 2003; Ulian, 2007). Usualmente é identificado um estímulo como antecedente, uma resposta operante e uma consequência, predominando antecedentes sociais, respostas verbais relacionadas a comportamentos-problema, seguidas por consequências aversivas.

A variabilidade de tipos de "antecedente", "resposta" e "consequência" desenhados sugere a dificuldade de o analista do comportamento analisar contingên- 
cias em sua atuação prática. Isso ocorre na medida em que são verificadas respostas ( $R$ ) no campos "antecedente" e "consequência", que são pontuadas emoções/ sentimentos/ sensações (E) na "resposta" e na "consequência" ou que são identificadas não-respostas (NR) no "antecedente", na "resposta" e na "consequência" (ver Figuras 1, 2 e 3). Tais achados sugerem confusão conceitual no uso clínico da teoria e são importantes na medida em que indicam onde é necessário investir mais esforços para expansão de conhecimento teórico, bem como treino na hora de educar os profissionais da área.

Ainda que na maioria das contingências avaliadas os autores desenhem no campo "antecedente" da contingência estímulos antecedentes (SANT) que estabelecem relação temporal imediata com a resposta a que se relacionam, e desenhem no campo "resposta" da contingência respostas operantes (O) ou respondente + operante $(\mathrm{R}+\mathrm{O})$, no preenchimento do campo "consequência" surpreendeu o número de contingências em que o autor não descreveu nenhum estímulo, o que sinaliza uma limitação bastante importante na elaboração das hipóteses sobre as variáveis relevantes do comportamento em questão.

Nessa direção, a avaliação feita de que 15\% das "consequências" desenhadas eram respostas (R) e $14 \%$ eram emoções/ sentimentos/ sensações (E) sugere problemas conceituais no uso da análise funcional, que tem sua finalidade prejudicada na medida em que não são identificadas as consequências que selecionaram e/ou mantêm determinada resposta, impactando na produção de resultados, entende-se, importantes para o andamento do caso.

Por outro lado, ainda que tenha havido dificuldade para identificar os estímulos consequentes relevantes à resposta em questão, quando estes são identificados (o que foi pontuado como SAV+, SAV-, SR+, SR- ou EXT), há um índice alto de estabelecimento de relação funcional (86\%). Isso, supõe-se, aumenta as chances de que a intervenção programada pelo terapeuta baseada na análise funcional desenvolvida tenha sucesso, quando apropriadamente identificadas as consequências mantenedoras da resposta em questão.

É possível que essa dificildade esteja relacionada ao uso predominante de relato verbal como única fonte dados em aproximadamente metade da amostra (seis dos 13 casos clínicos analisados). Isso porque esse limite no acesso aos múltiplos comportamentos pode desfavorecer a adequada operacionalização dos termos da contingência, perdendo precisão e impactanto a elaboração das intervenções e na mensuração dos resultados.

Outra possibilidade é que a variabilidade de $t i-$ pos de "antecedentes", "respostas" e "consequências" esteja associada a problemas teóricos. Por exemplo, no caso do desenho da emoção/sentimento/ sensação: onde devem se encaixados no modelo da contingência de três termos? Há espaço para isso? Como visto nos Resultados, por um lado foram raras as vezes em que os trabalhos selecionados colocaram um elemento a mais na contingência para identificar emoções, sentimentos e sensações. Por outro lado, nem sempre do ponto de vista teórico parece claro se, como e onde deveriam entrar as emoções, sentimentos e sensações na contingência. Contudo, na prática, os mais diversos desafios teóricos podem resultar em menor precisão no desenho da contingência.

A complexidade da transposição da teoria para a prática apareceu não somente na atuação do terapeuta como na condução desta pesquisa. Isso porque, em toda a amostra, o método empregado foi o relato de caso, confirmando a falta de estudos experimentais na área já mencionada por Leonardi (2016). Essa característica das pesquisas sugere que os terapeutas parecem atuar com base em hipóteses sobre possíveis variáveis de controle do comportamento-problema, de forma que, como afirma Ulian (2007), a validade interna pode ser questionada em decorrência do controle fraco sobre a intervenção adotada. Tais dados vão de encontro tanto com a recomendação de Sturmey et al. (2007) de utilizar o método experimental para a formulação de análise funcional, quanto com a concepção de que o delineamento A-B de caso único quase experimental seria o mais empregado na clínica (Ulian, 2007).

Destaca-se também que, frequentemente, o terapeuta não identifica o que faz ou, quando nomeia a prática, reconhece como sendo análise funcional. No entanto, isso ocorre sem haver diálogo claro com qual das distintas concepções teóricas a análise está alinhada e, portanto, com que tipo de prática profissional está relacionada, repercutindo em ainda mais distanciamento da teoria. 
Ainda que o presente trabalho possa contribuir para o avanço da área ao elucidar a falta de uniformidade no uso de análise funcional na terapia analítico-comportamental, alguns limites devem ser considerados. Como dito anteriormente, a ausência de uma metodologia de pesquisa clara e sistematizada na literatura sobre como fazer este tipo pesquisa faz com que o procedimento seja idiossincrático, sem possibilidade de replicação direta, enviesado pela história acadêmico-científico dos autores e permeado por dificuldades metodológicas inerentes aos dados coletados. Por exemplo, após a criação de categorias de coleta e de análise, o Método teve que ser revisto durante a coleta, uma vez que as palavras que os autores dos casos utilizaram nos "antecedentes", "respostas" e "consequências", alternando entre substantivos e verbos, prejudicou a categorização. Em alguns casos, o uso de verbos na consequência sugeria que tratava-se de uma ação/resposta e não de um estímulo, por exemplo. Espera-se que novas pesquisam possam contribuir com modos de sistematização dos dados sobre o uso da análise funcional na terapia analítico-comportamental.

Por fim, é importante relembrar que todos os textos examinados neste trabalho eram brasileiros, o que, portanto, revela a forma de fazer análise funcional mais utilizada neste país (cf. Toscano, Macchione \& Leonardi, 2019). Entretanto, diversos modelos de análise funcional podem ser encontrados na literatura comportamental. Por exemplo, Sturmey (2008), em seu livro Behavioral Case Formulation and Intervention: A Functional Analytic Approach, apresenta mais de 10 diferentes tipos de análise funcional, apontando que eles "compartilham alguns elementos comuns e também diferem de várias maneiras significativas" ( $p$. 222). Além disso, determinadas modalidades de terapia comportamental desenvolveram seus próprios modelos de análise funcional, como é o caso da terapia comportamental dialética (cf. Leonardi, 2018). Nesse sentido, o desafio atual parece ser alcançar algum grau de unificação conceitual no campo da análise funcional.

Conclui-se, com base nos dados atuais, que as dificuldades se estendem do modo de produção de conhecimento para a prática. Como afirma Skinner (1953/1965), “confusão na teoria significa confusão na prática" (p. 10). Portanto, entende-se que, conforme o conhecimento científico e profissional for se aprimorando, a prática deve ficar cada vez mais refinada. Para isso, o esforço de pesquisadores e terapeutas se faz necessário, com novos trabalhos e ampliação dos conhecimentos atuais tanto em prol de refinamento teórico, quanto de aplicações práticas publicadas com o devido rigor metodológico.

\section{Referências}

Andery, M. M. P. A., Micheletto, N. \& Sério, T. M. A. P. (2001). Análise funcional na análise do comportamento. Em H. J. Guilhardi (Org.), Sobre comportamento e cognição: Expondo a variabilidade (Vol. 8, pp. 148-157). Santo André: Esetec.

Borges, N. B. (2010). Discutindo o atentar como comportamento precorrente na clínica analítico-comportamental: extendendo a avaliação funcional. Em M. R. Garcia, P. R. Abreu, E. N. P. Cillo, P. B. Faleiros \& P. Piazzon (Orgs.), Sobre comportamento e cognição: Terapia comportamental e cognitiva (Vol. 27, pp. 367-378). Santo André: Esetec.

Costa, N. (2011). O surgimento de diferentes denominações para terapia comportamental no Brasil. Revista Brasileira de Terapia Comportamental e Cognitiva, 13(2), 46-57. doi: 10.31505/rbtcc.v13i2.453

Haynes, S. N. \& O'Brien, W. H. (1990). Functional analysis in behavior therapy. Clinical Psychology Review, 10, 649-668. doi: 10.1016/02727358(90)90074-K

Lazarus, A. (1980). Terapia multimodal do comportamento. Atualização em terapia do comportamento (Vol. 1). São Paulo: Manole.

Leonardi, J. L. (2016). Prática baseada em evidências em psicologia e a eficácia de análise do comportamento clínica (Tese de doutorado). Universidade de São Paulo, São Paulo, SP.

Leonardi, J. L. (2018). A terapia comportamental dialética (DBT). Em A. E. A. Antúnez \& G. Safra (Orgs.), Psicologia clínica: da graduação à pós-graduação (pp. 95-103). São Paulo: Atheneu. 
Leonardi, J. L., Borges, N. B. \& Cassas, F. A. (2012). Avaliação funcional como ferramenta norteadora da prática clínica. Em N. B. Borges \& F. A. Cassas (Orgs.), Clínica analítico-comportamental: Aspectos teóricos e práticos (pp. 105-109). Porto Alegre: Artmed.

Neno, S. (2003). Análise funcional: definição e aplicação na terapia analítico-comportamental. Revista Brasileira de Terapia Comportamental e Cognitiva, V, 151-165.

Skinner, B. F. (1965). Science and human behavior. New York: Free Press. (Trabalho original publicado em 1953)

Sturmey, P. (2008). Behavioral case formulation and intervention: A functional analytic approach. Chichester: John Wiley \& Sons.

Sturmey, P., Ward-Horner, J., Marroquin, M. \& Doran, E. (2007). Structural and functional approaches to psychopathology and case formulation. Em P. Surmey (Org.), Functional analysis in clinical treatment (pp. 1-22). Burlington: Elsevier.

Toscano, M. P., Macchione, A. C. C. \& Leonardi, J. L. (2019) Análise funcional do comportamento. Em W. V. Melo. (Org.), A prática das intervenções psicoterápicas: como tratar pacientes na vida real (pp. 84-101). Novo Hamburgo: Sinopsys.

Ulian, A. L. A. O. (2007). Uma sistematização da prática do terapeuta analítico-comportamental: Subsídios para formação (Tese de doutorado). Universidade de São Paulo, São Paulo, SP.

Virués-Ortega, J. \& Haynes, S. N. (2005). Functional analysis in behavioral therapy. International Journal of Clinical and Health Psychology, 5, 567-587.

Yoman, J. (2008). A primer on functional analysis. Cognitive and Behavioral Practice, 15, 325-340. doi: 10.1016/j.cbpra.2008.01.002

\section{Apêndice: lista dos trabalhos que compuseram a amostra}

Arruda, M. C. C. \& De-Farias, A. K. C. R. (2009). Multideterminação de uma disfunção sexual: um breve estudo de caso. Em R. C. Wielenska (Org.), Sobre comportamento e cognição: Desafios, soluções e questionamentos (Vol. 24, pp. 183-188). Santo André: Esetec.

Batista, M. P. S. \& Ribeiro, M. R. (2008). Comportamento governado por regras e resistência às mudanças: um estudo de caso. Em W. C. M. P. Silva (Org.), Sobre comportamento e cognição: Análise comportamental aplicada (Vol. 21, pp. 121-134). Santo André: Esetec.

Bueno, G. N. (2009). Quando as obsessões-compulsões interditam a vida: a intervenção pelas estratégias comportamentais. Em R. C. Wielenska (Org.), Sobre comportamento e cognição: Desafios, soluções e questionamentos (Vol. 23, pp. 346-360). Santo André: Esetec.

Elias, P. V. O. (2005). Terapia comportamental aplicada ao tratamento da obesidade. Em H. J. Guilhardi \& N. C. Aguirre (Orgs.), Sobre comportamento e cognição: Expondo a variabilidade (Vol. 16, pp. 164-173). Santo André: Esetec.

Ferreira, L. N. S. \& De-Farias, A. K. C. R. (2009). Autoconhecimento e relações amorosas: um estudo de caso. Em R. C. Wielenska (Org.), Sobre comportamento e cognição: Desafios, soluções e questionamentos (Vol. 24, pp. 56-64). Santo André: Esetec.

Guilhardi, H. J. \& Oliveira, W. (2001). Linha de base múltipla: possibilidades e limites deste modelo de controle de variáveis em situação clínica. Em R. A. Banaco (Org.), Sobre comportamento e cognição: Aspectos teóricos, metodológicos e de formação em análise do comportamento e terapia cognitivista (Vol. 1, pp. 348-384). Santo André: Esetec. (Trabalho original publicado em 1997)

Guilhardi, H. J. \& Queiroz, P. B. P. S. (2001). A análise funcional no contexto terapêutico o comportamento do terapeuta como foco da análise. Em M. Delitti (Org.), Sobre comportamento e cognição: A prática da análise do comportamento e da terapia cognitivo comportamental (Vol. 2, pp. 45- 97). Santo André: Esetec. (Trabalho original publicado em 1997) 
Martins, L. J. \& Guilhardi, H. J. (2006). História de contingências coercitivas e suas implicações: Estudo de caso sob a perspectiva da Terapia por Contingências de Reforçamento (TCR). Em H. J. Guilhardi \& N. C. Aguirre (Orgs.), Sobre comportamento e cognição: Expondo a variabilidade (Vol. 17, pp. 231-259). Santo André: Esetec.

Meneghelo, M. H. B. G., Neto, A. A \& Teixeira, M. C. T. V. (2005). Autoconhecimento: uma via de mão dupla entre terapeuta e cliente. Em H. J. Guilhardi \& N. C. Aguirre (Orgs.), Sobre comportamento e cognição: Expondo a variabilidade (Vol. 15, pp. 428-441). Santo André: Esetec.

Moriyama, J. S. (2007). Processo terapêutico analítico-comportamental em dois casos de transtorno dismórfico corporal (Tese de doutorado). Pontifícia Universidade Católica de Campinas, Campinas, SP.

Rocha, J. F. (2012). Efeitos de uma intervenção comportamental com treino de habilidades sociais para universitários com fobia social (Dissertação de mestrado). Universidade Estadual Paulista Júlio de Mesquita Filho, Bauru, SP.

\section{Informações do Artigo}

Histórico do artigo:

Submetido em: 13/06/2018

Primeira decisão editorial: 08/02/2019

Versão definitiva aceita em: 17/04/2019

Editor Associado: Denis Roberto Zamignani 